understood), and by their happy choice of Prof. M. S. Bartlett as general editor of the series.

In the monograph reviewed here Prof. P. A. P. Moran of the Australian National University, Canberra, writes on the theory of storage systems in which either the input or the output is of stochastic character. Most space is given to problems of the first type ("The Theory of Dams") ; here a random input of water feeds a reservoir or a system of reservoirs subject to overflow and release rules of various types, and one is interested both in the conditions for statistical equilibrium and the statistical distributions which characterize it, and also in transient phenomena such as the risk that the reservoir will run dry within a given time period when the initial conditions are known. This is a very recent branch of applied probability, and can be said to be Prof. Moran's peculiar creation, for apart from some exceedingly interesting pioneer work by Hurst practically all the mathematical papers devoted to the subject have been written by Moran himself or by those influenced by him. The programme of research opened up by Moran in 1954 is still very far from complete; much more remains to be done on systems with a (random) continuous input, very little is known yet about the behaviour of networks of reservoirs, and a satisfactory treatment of correlated inputs is still lacking (as it is also in queueing theory, although recent work by Dagnall, Winsten, and Wishart has shown that significant progress can be made if the problem is suitably formulated). Those familiar with the earlier literature will be very grateful to the author for this timely survey of what has been done and of what remains to be done; others will be equally grateful for the possibility which this book offers them of joining in a very active programme of research.

D. G. KENDALL

\section{QUANTUM EFFECTS ON A MACROSCOPIC SCALE}

Liquid Helium

By Prof. K. R. Atkins. (Cambridge Monographs on Physics.) Pp. $x+312$. (Cambridge: At the University Press, 1959.) 60s. net.

$\mathrm{T}$

HE strange behaviour of liquid helium at low temperatures has been the subject of much work and thinking for some time. Ever since Kamerlingh Onnes succeeded in liquefying helium some fifty years ago, work has been going on with this single substance ; more and more new phenomena have been discovered and a new field of experiments and theory has been established. At first, the difficult techniques for obtaining the necessary low temperatures meant that the work was concentrated in a few laboratories which became famous in this field. With the more recent methods these low temperatures are easier to obtain, and much work is going on in many places all over the world. The subject is still in rapid development. A new monograph which tries to select the essential features in such a vast field of experiments and theoretical thinking is very welcome. At the same time, it is a somewhat bold enterprise to write a monograph on such a rapidly progressing subject. A few points were already out of date by the time that the book was printed.

The wide variety of strange phenomena connected with liquid helium led slowly towards a pattern of basic facts. Some of these are by now essentially understood, others are not, and many more are perhaps still to be discovered. Theoretical ideas and experimental discoveries proceeded hand in hand in a fruitful co-operation. There are many striking examples of this interaction between theory and experiment. The concept of elementary excitations which move around in the superfluid background was introduced by Landau and is essential for the understanding of many properties of liquid helium. These excitations, phonons and rotons, characterize the low-lying excited states of the system. Landau's predictions about the excitation spectrum have been recently confirmed by neutron-scattering experiments. The elementary excitations form the 'normal component' of the liquid, which together with the superfluid component leads to a 'two-fluid' theory from which many features of the hydrodynamic and thermal behaviour of liquid helium can be understood. Other features which are less well understood are related to the formation of vortices. According to the ideas of Onsager and Feynman, the strength of these vortices has quantized values, a prediction which has found experimental verification in the past few years.

Quantum properties play an important part in the whole behaviour of liquid helium, and some manifestations of these are on a macroscopic scale. The Bose statistics obeyed by the helium-4 atoms seems also to be essential for the superfluid behaviour of helium. The helium-3 isotope which obeys Fermi statisties does not show superfluidity. Though many features of liquid helium are understood, much progress is still needed in a detailed theoretical description. The phenomena are related to the collective behaviour of a large number of particles, and the wave mechanics of such a system is of considerable mathematical complexity. But a better understanding of the collective behaviour of helium atoms may be of significance also in connexion with other similar problems of physies.

The book under review succeeds in a fair selection. of the relevant experimental facts in making a good systematic treatment. This will be of value both to the expert and for reference. Some parts in the theoretical sections are perhaps not of the same standard, and especially the author's estimate of the theoretical achievements seems to be slightly overoptimistic. The book covers a lot of ground, it makes pleasant reading, and will be enjoyed not only by the specialists but also by those who look merely for orientation in this rather exciting subject.

$$
\text { J. G. Valatin }
$$

\section{FRESHWATER INVERTEBRATES}

\section{A Guide to Freshwater Invertebrate Animals}

By Dr. T. T. Macan. Pp. $\mathrm{x}+118$. (London: Longmans, Green and Co., Ltd., 1959.) $11 s .6 d$. net.

7 HE importance of freshwater biology for scientific, economic and educational purposes has greatly increased in recent years and the many biologists, both amateur and professional, now interested in the subject, will be grateful to T. T. Macan for writing an attractive, straightforward and well-illustrated "Guide to Freshwater Invertebrate Animals".

The line drawings, of which there are more than two hundred, are simple, clear and attractively 